Filippo Cademartiri

Ludovico La Grutta

Giuseppe Runza

Alessandro Palumbo

Erica Maffei

Nico RA Mollet

Tommaso Vincenzo Bartolotta

Pamela Somers

Michiel Knaapen

Stefan Verheye

Massimo Midiri

Ronald Hamers

Nico Bruining

Received: 16 May 2006

Revised: 28 September 2006

Accepted: 17 November 2006

Published online: 24 January 2007

(C) Springer-Verlag 2007

F. Cademartiri $(\bowtie) \cdot$ L. La Grutta •

G. Runza $\cdot$ A. Palumbo $\cdot$ N. R. Mollet .

R. Hamers · N. Bruining

Department of Radiology,

Erasmus Medical Center,

Dr. Molenwaterplein, 40,

3015GD Rotterdam, The Netherlands

e-mail: filippocademartiri@hotmail.com

Tel.: +31-010-4634127

Fax: +31-010-4634033

F. Cademartiri · L. La Grutta •

G. Runza - A. Palumbo - N. R. Mollet

R. Hamers · N. Bruining

Department of Cardiology,

Erasmus Medical Center,

Rotterdam, The Netherlands

F. Cademartiri · A. Palumbo - E. Maffei

Department of Radiology,

Azienda Ospedaliero-Universitaria,

Parma, Italy

F. Cademartiri - A. Palumbo - E. Maffei

Department of Cardiology,

Azienda Ospedaliero-Universitaria,

Parma, Italy

\title{
Influence of convolution filtering on coronary plaque attenuation values: observations in an ex vivo model of multislice computed tomography coronary angiography
}

L. La Grutta · G. Runza •

T. V. Bartolotta $\cdot$ M. Midiri

Department of Radiology, DIBIMEL,

University of Palermo,

Palermo, Italy

P. Somers · M. Knaapen

Department of Pathology,

Middelheim Hospital,

Antwerp, Belgium

S. Verheye

Department of Cardiology,

Middelheim Hospital,

Antwerp, Belgium

Abstract Attenuation variability (measured in Hounsfield Units, HU) of human coronary plaques using multislice computed tomography (MSCT) was evaluated in an ex vivo model with increasing convolution kernels. MSCT was performed in seven ex vivo left coronary arteries sunk into oil followingthe instillation of saline $(1 / \infty)$ and a $1 / 50$ solution of contrast material $(400 \mathrm{mgI} / \mathrm{ml}$ iomeprol). Scan parameters were: slices/ collimation, $16 / 0.75 \mathrm{~mm}$; rotation time, 375 ms. Four convolution kernels were used: b30f-smooth, b36fmedium smooth, b46f-medium and b60f-sharp. An experienced radiologist scored for the presence of plaques and measured the attenuation in lumen, calcified and noncalcified plaques and the surrounding oil. The results were compared by the ANOVA test and correlated with Pearson's test. The signal-to-noise ratio (SNR) and contrast-to-noise ratio (CNR) were calculated. The mean attenuation values were significantly different between the four filters $(p<0.0001)$ in each structure with both solutions. After clustering for the filter, all of the noncalcified plaque values $(20.8 \pm 39.1,14.2 \pm 35.8,14.0 \pm 32.0$, $3.2 \pm 32.4 \mathrm{HU}$ with saline; $74.7 \pm 66.6$, $68.2 \pm 63.3,66.3 \pm 66.5,48.5 \pm 60.0 \mathrm{HU}$ in contrast solution) were significantly different, with the exception of the pair b36f-b46f, for which a moderatehigh correlation was generally found. Improved SNRs and CNRs were achieved by b30f and b46f. The use of different convolution filters significantly modifief the attenuation values, while sharper filtering increased the calcified plaque attenuation and reduced the noncalcified plaque attenuation.

Keywords Multislice computed tomography - Coronary angiography . Coronary plaque $\cdot$ Convolutions kernels 


\section{Introduction}

Multislice computed tomography (MSCT) of the coronary arteries is becoming an established method to exclude the presence of significant stenosis because of the high negative predictive value [1-6]. Stenosis, however, occurs in a later stage of coronary artery disease (CAD); consequently, the atherosclerotic plaque composition and morphology may represent a better predictor of plaque stability [7-9]. Until recently, only invasive techniques, such as intracoronary ultrasound (ICUS), have been able to provide consistently reproducible quantitative and qualitative information on plaque composition [10]. ICUS, however, cannot be used for routine evaluation of plaque characteristics because of its invasiveness and related increased risk, additional time and cost.

Magnetic resonance imaging may be used to characterize in vivo the atherosclerotic plaque morphology and composition in carotid [11] and coronary arteries [12, 13].

Several recent in vivo studies have stressed that MSCT can be used to characterize coronary atherosclerotic plaque and has the potential to become the noninvasive modality of choice for plaque imaging [14-17]. This technique is able to provide information on noncalcified coronary plaques, which may be useful to identify a high-risk plaque [18-20].

MSCT plaque assessment is based upon the variable $\mathrm{X}$-ray attenuation of the tissue components. However, several parameters, such as lumen attenuation, convolution filtering, body mass index of the patient and contrast-tonoise ratio (CNR) of the images, are able to modify the attenuation values that are being used to define the composition of coronary plaques.

The aim of the present study was to investigate the effect of increasing convolution filtering on plaque components with varying coronary attenuation in an ex vivo coronary model.

\section{Material and methods}

\section{Specimens}

Seven ex vivo left anterior descending (LAD) coronary arteries were obtained at autopsy (Fig. 1) from seven deceased patients: four male and three female, with a mean age of $68 \pm 10$ years: five (four males and one female with an age between 62 and 71 years) jad died of noncardiovascular diseases, and two (one male and one female with an age of 65 and 73 years, respectively) had died of ischemic heart disease. The Institutional Review Board approved the study protocol.

The specimens were prepared and examined separately. Each coronary artery was fitted with two cannulas fixed with surgical thread in the proximal (in the left main) and distal ends (in the LAD). The circumflex artery had been closed earlier at its end with thread. Only the LAD was used because of the major length (segments considered

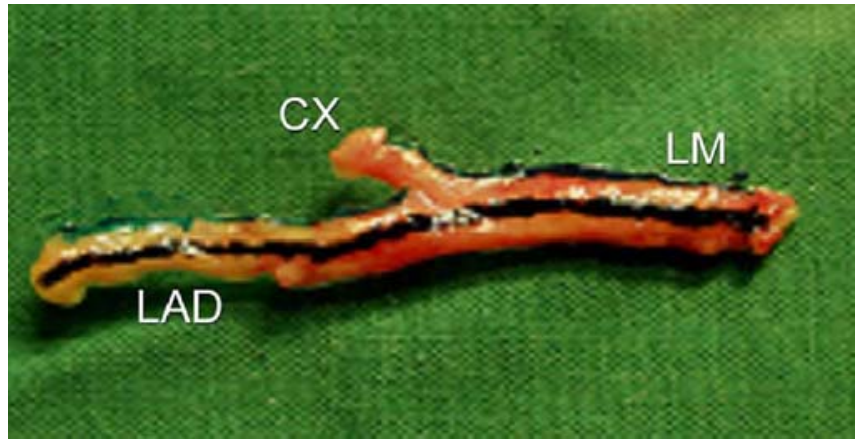

Fig. 1 Example of proximal left coronary artery specimen. $C X$ Left circumflex, $L A D$ left anterior descending artery, $L M$ left main coronary artery

with a mean length of $7.57 \mathrm{~cm}$ ) and higher prevalence of atherosclerotic disease and for technical reasons (ability to fix a surgical thread in the left main, without hampering the evaluation of the proximal LAD).

\section{Contrast material}

Two solutions were used: a saline and a 1/50 dilution of contrast material $(400 \mathrm{mgI} / \mathrm{ml}$ Iomeprol; Bracco, Italy). The attenuation values (Hounsfield Units, HU) of the two solutions, measured in a $10-\mathrm{ml}$ syringe after dilution, were $15.9 \pm 1.8 \mathrm{HU}(1 / \infty$; defined as saline; no contrast material was diluted) and $414.8 \pm 5.6 \mathrm{HU}(1 / 50$; defined as contrast solution). We preferred to use a $1 / 50$ dilution to achieve a mean coronary in-lumen density $>300 \mathrm{HU}-$ which is not typically achieved in in vivo cardiac computed tomography angiography (CTA) studies - in order to emphasize the difference with the saline solution. Our study benefits from two consecutive scans of the same vessel (e.g. with and without contrast media) for a comparative evaluation of plaques, which is not truly feasible during in vivo studies due to high radiation exposure.

\section{Experimental settings}

A box was filled with olive oil. Prior to positioning the specimen in the oil, saline was injected through the sheaths to wash out the air bubbles in the lumen as much as possible.

Once the specimen had sunk into the oil, which simulated epicardial fat, the solution was instilled through the sheaths using a 10-ml syringe from the proximal end of the specimen. The injection was finished when the solution was observed leaking out of the distal end of specimen. The leaking solution was removed from the specimen using a syringe. The same procedure was used to fill the LAD with both solutions. The specimen was kept for all investigations in the same longitudinal position (head-LM-to feetdistal end of LAD ) to obtain direct cross-section images. 


\section{Scan parameters}

A MSCT scan (Somatom Sensation 16; Siemens, Germany) was performed following the intra-coronary injection of two solutions.

Scans were performed at the following parameters: slices/collimation, 16/0.75 mm; rotation time, $375 \mathrm{~ms}$; feed per rotation $3.0 \mathrm{~mm}$ (pitch: $0.25 \mathrm{~mm}$ ); $120 \mathrm{kv} ; 400 \mathrm{mAs}$; effective slice thickness, $1 \mathrm{~mm}$; reconstruction increment, $0.5 \mathrm{~mm}$; field of view (FOV), $100 \mathrm{~mm}$. Four convolution filters were used: b30f (smooth), b36f (medium smooth), b46f (medium) and b60f (sharp). Based on our clinical experience in routine evaluation, b30f was used instead of b20f because of a better delineation of the coronary arteries and atherosclerotic lesions.

The scan geometry was based on a retrospective electrocardiogram (ECG)-gated protocol (the same used for the in vivo examination). This protocol is based on a low pitch that allows a retrospective reconstruction of multiple phases within the cardiac cycle. A demo-ECG was switched on and a heart rate of 71 occurred during the scan. The half-rotation reconstruction algorithm brought the effective temporal resolution down to $187 \mathrm{~ms}$.

\section{Data collection and analysis}

A radiologist with a level 3 expertise in cardiac CT [21] loaded the data sets into a dedicated workstation (Leonardo; Siemens, Germany) and performed orthogonal views of all specimens for each convolution filter with two solutions.

One operator performed all of the measurements. Each specimen was evaluated for the presence of atherosclerotic plaque. The coronary wall thickening of plaque (minimum size observed: $0.3 \mathrm{~mm}^{2}$ ) was clearly visible from the surrounding oil with low attenuation and from the lumen after injection of contrast material. Plaques were targeted regardless of their size. The operator loaded the two data sets for each solution of the same specimen into a workstation screen, scrolling the data sets in parallel with standard softtissue window settings (window width:700 HU; window center:140 HU).

Once a plaque was detected in the two solutions by means of a comparative evaluation, the operator drew four regions of interest (ROIs) for the contrast material: in the lumen of the vessel (defined as lumen), the soft tissue of the coronary plaque (defined as noncalcified plaque), the calcification within the coronary artery wall (defined as calcified plaque) and the oil surrounding the plaque (defined as surrounding). The ROIs (minimum size: $0.1 \mathrm{~mm}^{2}$ ) were drawn as large as possible while avoiding the borders of each structure in order to limit the partial volume effect. Once the four ROIs were drawn, the operator could copy and paste these into other stacks of images. The two data sets for each solution were then evaluated using four convolution filters (b30f, b36f, b46f and b60f). For each ROI the mean and the standard deviation of the attenuation value were collected. Overall, 3200 attenuation values were obtained.

\section{Statistical analysis}

The attenuation values are presented as means and standard deviations. The signal-to-noise ratio (SNR) was calculated as the mean attenuation value of the ROI/standard deviation of the surrounding oil attenuation value. The standard deviation $\mathrm{HU}$ value of the surrounding air is often used for the estimation of noise. However, we did not include the air in our images. The standard deviation of the surrounding oil can estimate the noise because of its reliable signal. SNRs are presented as means for each kernel.

The contrast-to-noise ratio (CNR) was calculated as (mean attenuation $\mathrm{A}$ - mean attenuation $\mathrm{B}$ )/image noise (defined as the standard deviation of the surrounding oil). CNRs of (lumen - noncalcified plaque), (noncalcified plaque - surrounding), (calcified plaque - noncalcified plaque) and (calcified plaque - lumen) were calculated and presented as average for each kernel and solution.

Statistical evaluation was performed with dedicated software (SPSS ver. 10.1; SPSS, Chicago, Ill.). A one-way ANOVA test was performed to analyze the statistical difference between kernels in each structure for two solutions. A $p<0.0001$ was considered for statistical significance. The Tukey HSD test evaluated the statistical absolute difference between any two groups of values. The Pearson's correlation was also tested between all the different convolution filters.

After clustering for kernel and structure, the attenuation values obtained with the two solutions were compared with Student's $t$-test and correlated with Pearson's test.

\section{Results}

A total of 100 sections containing mixed plaques $(n=20)$ were evaluated in the seven coronary specimens. Two solutions and four convolution kernels were available $(800$ slices) at each level, and four ROIs (lumen, surrounding, calcified plaque, noncalcified plaque) were sampled in each slice. A total of 3200 measurements were performed. The results are summarized in Table 1 . The attenuation values were all significantly different between the convolution filters in each structure with both solutions $(p<0.0001)$. The results of the Tukey HSD to test the difference between pairs of four filters showed that the noncalcified plaque attenuation values were all significantly different using both solutions $(p<0.01)$, with the exception of pair b36f-b46f. The attenuation values between b30f and b46f were significantly different at a high concentration in calcified plaques, whereas for the same pair of filters the attenuation values of the lumen and the surrounding were not significantly different. After 
Table 1 Summary of the attenuation values measured in each structure in the two solutions (e.g. saline and contrast solutions) with increasing convolution filter

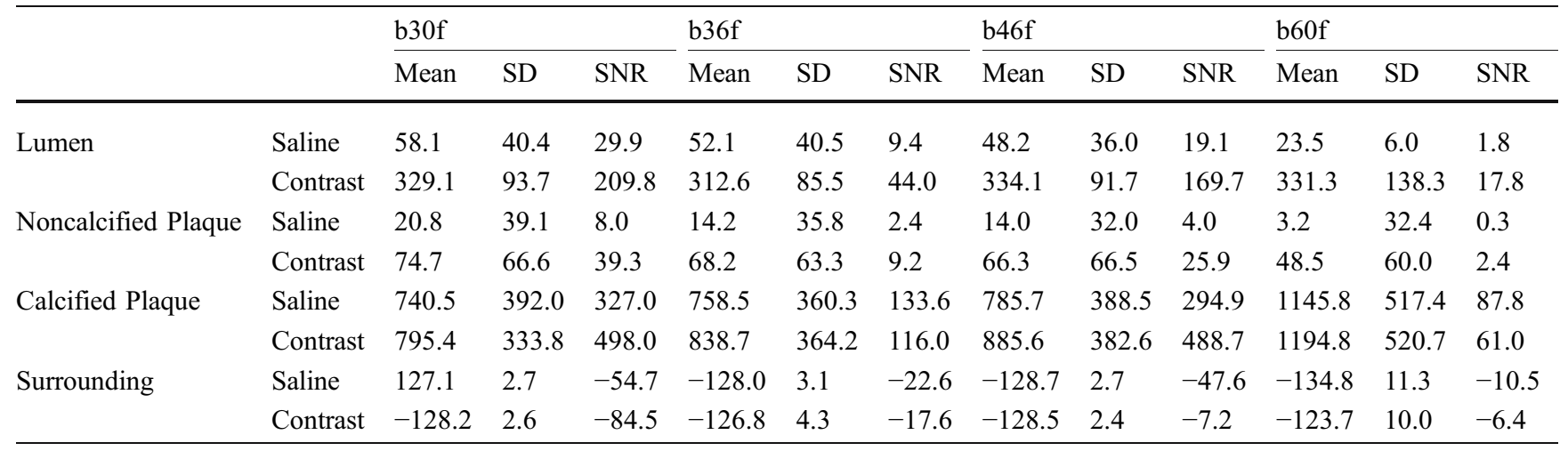

clustering the paired attenuation values of the four structures for the convolution filter, a moderate-high correlation was generally found, with the exception of pairs b30f-b46f in surrounding $(r=0.150), \mathrm{b} 30 \mathrm{f}-\mathrm{b} 60 \mathrm{f}$ in lumen $(r=0.206)$ and surrounding $(r=0.311)$, b36f-b60f in lumen $(r=0.132)$ and b46f-b60f $(r=0.201)$ in lumen in the saline solution.

Whereas the convolution filter did not significantly affect the attenuation values of the lumen and the surrounding, sharper filters decreased the attenuation of the plaque and, conversely, increased the attenuation of the calcification with both solutions (Fig. 2).

The SNR values appear to have a common trend with increasing convolution filtering and both solutions. However, the best SNR was achieved with the b30f and b46f filters. Plaque values follow the lumen values, while the surrounding and calcification patterns are similar (Fig. 3).
The CNR values were calculated between structures with increasing convolution filters and different solutions (Table 2). Improved CNRs were achieved with the b30f and b46f filters. The lumen-noncalcified plaque CNR was particularly improved with the b30f and b46f filters using a high solution of contrast media. The noncalcified plaquesurrounding CNR was improved with the b30f and b46f filters in contrast solution. Calcified plaque-noncalcified plaque CNR increased with the contrast solution with the b30f and b46f filters, while it decreased using the b36f and b60f filters. The b46f filter was found to be ideal for calcified plaquelumen contrast since it improved the CNR at contrast solution.

After clustering the paired attenuation values obtained for the four structures in order to test the statistical difference between saline and contrast solution for each convolution filter, values were all significantly different
Fig. 2 Mean attenuation of the four structures in the two solutions (e.g. saline and contrast) with increasing convolution filters. Whereas the convolution filter does not significantly affect the attenuation values of the lumen and the surrounding, sharper filters decrease the attenuation of the noncalcified plaque and, conversely, increase the attenuation of the calcified plaque with both solutions
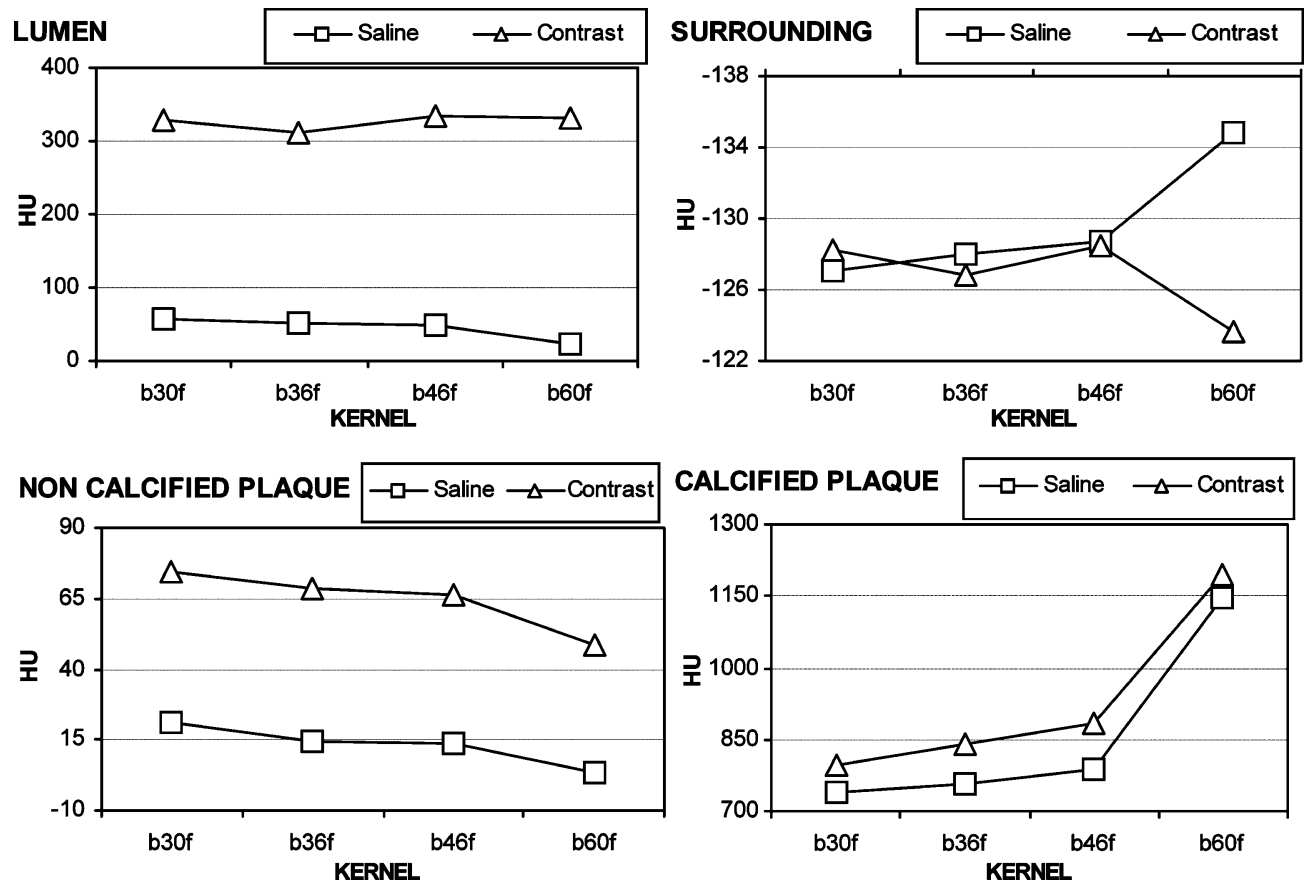
Fig. 3 The SNR values appear to have a common trend with increasing convolution filtering and both solutions (e. g. saline and contrast). However, the best SNR is achieved with b30 and b46 filters. Noncalcified plaque values follow the lumen values, while the surrounding and the calcified plaque patterns are similar
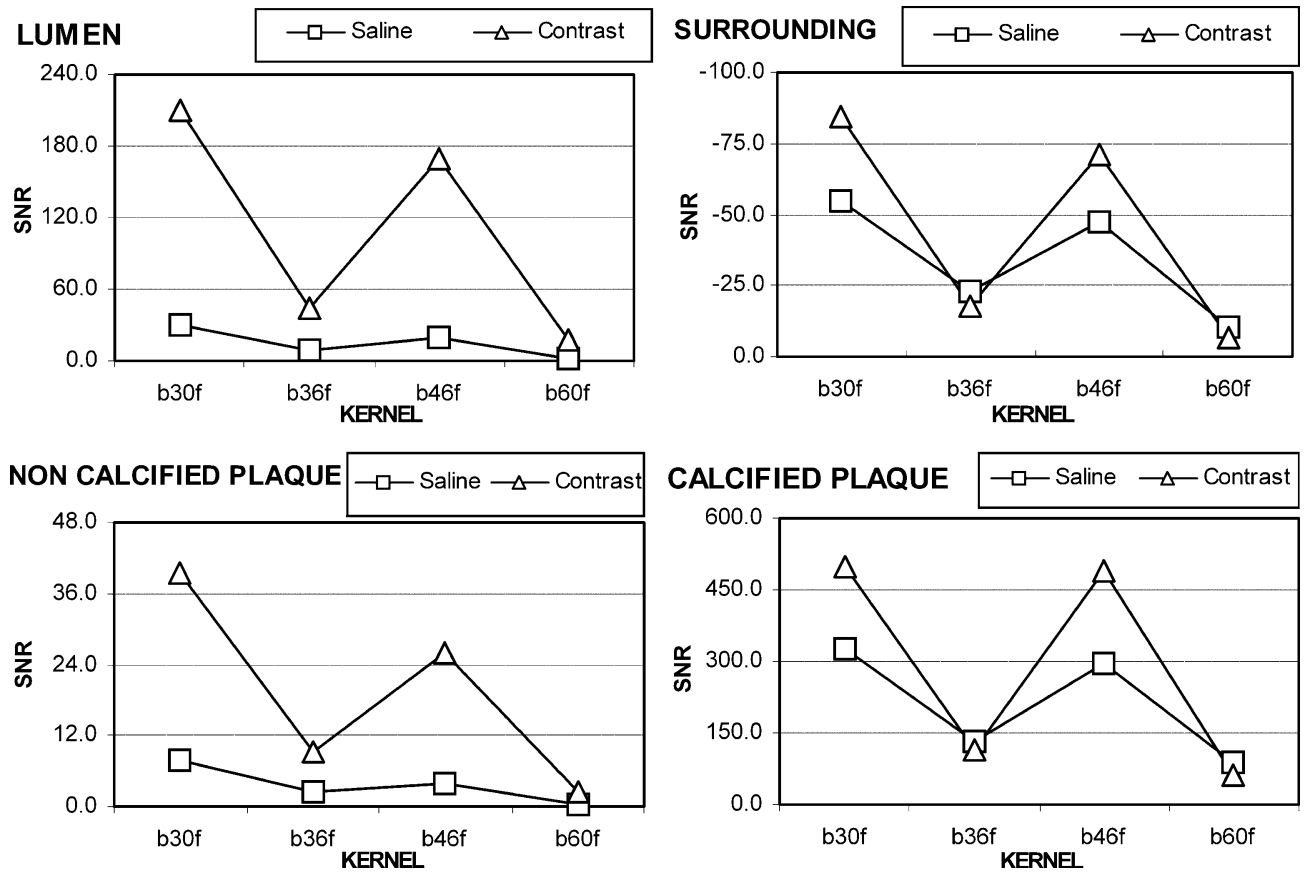

$(p<0.05)$ apart from surrounding with $\mathrm{b} 46 \mathrm{f}$ and calcification with b60f. Pearson's test has shown good correlation only between the attenuation values of calcification $(r=0.738, \quad r=0.826, r=0.759, r=0.723$ with b30f, b36f, b46f, b60f, respectively).

\section{Discussion}

The evaluation of the coronary artery wall is a data set that has become available since the introduction of coronary MSCT. Several studies have reported the ability of MSCT to detect coronary plaques and subsequently characterize their tissue composition (i.e. calcified, mixed, noncalcified) $[14,20]$. In an in vivo study, Kopp et al. showed an excellent correspondence between ICUS criteria (soft, intermediate and calcified plaques) and MSCT attenuation values [14]. Schroeder et al. subsequently found a strong correlation between tissue density measurements within the plaque and the qualitative ICUS criteria. The CT attenuation results were $14 \pm 26 \mathrm{HU}$ for plaques classified by ICUS as soft (i.e. predominantly lipid) plaques, $91 \pm 21 \mathrm{HU}$ for plaques classified by ICUS as intermediate (i.e. predominantly fibrous) plaques and $419 \pm 194 \mathrm{HU}$ for plaques classified by ICUS as calcified. These researchers reported no overlap in the mean attenuation among the three groups [15]. Leber et al. showed that lesion echogenity correlated well with MSCT attenuation measurements in coronary plaque, with MSCT correctly classifying $78 \%$ of sections containing hypoechoic plaque areas (soft plaques), $78 \%$ of sections containing hyperechoic plaque areas (fibrous plaques) and $95 \%$ of sections containing calcified plaque tissue. The MSCT density measurements within coronary lesions revealed significantly different values for hypoechoic, hyperechoic, and calcified plaques [17]. The close correlation between ICUS

Table 2 Summary of CNR values calculated between structures with increasing convolution filters and different solutions

\begin{tabular}{|c|c|c|c|c|c|}
\hline & & b30f & b36f & b46f & b60f \\
\hline \multirow[t]{4}{*}{ Saline } & Lumen - noncalcified plaque & 21.8 & 7.1 & 15.1 & 1.5 \\
\hline & Non - calcified plaque-surrounding & 62.7 & 25.0 & 51.6 & 10.8 \\
\hline & Calcified plaque - noncalcified plaque & 318.9 & 131.3 & 290.8 & 87.4 \\
\hline & Calcified plaque - lumen & 297.1 & 124.2 & 275.7 & 85.9 \\
\hline \multirow[t]{4}{*}{ Contrast solution } & Lumen - noncalcified plaque & 170.6 & 34.8 & 143.8 & 15.4 \\
\hline & Noncalcified plaque - surrounding & 123.7 & 26.9 & 97.1 & 8.8 \\
\hline & Calcified plaque - noncalcified plaque & 458.7 & 106.7 & 462.8 & 58.5 \\
\hline & Calcified plaque - lumen & 288.2 & 71.9 & 319.0 & 43.1 \\
\hline
\end{tabular}


and MSCT in terms of the detection of calcified and noncalcified coronary plaques was also shown by Achenbach et al., although this group found a sensitivity of only $53 \%$ for exclusively noncalcified plaque [16].

Becker et al. compared the atherosclerotic lesions of 11 human cadaver heart specimens detected by MSCT with the histopathological macroscopic findings according to American Heart Association criteria. They concluded that MSCT is a promising tool for the characterization of atherosclerotic coronary plaques [22].

Such information is of great value since the risk of acute coronary syndromes caused by plaque disruption and thrombosis depends on plaque composition (i.e. noncalcified, predominantly lipid plaques with positive remodeling) rather than stenosis severity [18-20]. Reliable and reproducible non-invasive methods for the assessment of plaque constitution could be important in risk stratification of patients with CAD.

Nevertheless, several aspects (i.e. the impact of lumen attenuation, convolution filters, body mass index of the patient, CNR of the images and coronary calcification) of the methodology need to be better addressed in order to validate the accuracy of attenuation values measured within the coronary plaques using MSCT.

It is controversial if the absolute HU values are valid measurements for determining the plaque composition and identifying the corresponding tissue. A phantom study revealed that intracoronary attenuation can significantly affect the measured plaque attenuation so that accurate plaque tissue differentiation may be influenced [23]. Furthermore, Cademartiri et al. recently reported that the intravascular attenuation modifies significantly the attenuation of the coronary atherosclerotic plaques in an ex vivo coronary specimen [24]. Therefore, the characterization of the plaque on the basis of absolute attenuation values should be reported with caution, and the intraluminal attenuation should also be reported. The calibration of tissue attenuation values with contrast in-lumen values may improve accurate tissue component identification.
New possibilities for coronary artery plaque imaging may be offered by dual-source CT, which provides robust diagnostic image quality independent of the heart rate. Potential applications of dual-energy CT include tissue characterization and $\mathrm{Ca}$ quantification of atherosclerotic plaques $[25,26]$.

In our experimental study we addressed the issue of the variability of coronary plaque attenuation by varying the convolution filters (Fig. 4).

The direct back projection of CT attenuation profiles results in unsharp images. To prevent unsharp and approximate images, each projection has to be convolved with a predetermined mathematical function, the convolution kernel; this kernel represents a filtering procedure that is able to enhance or reduce object boundaries. Convolution influences image characteristics by the choice and design of the kernel (i.e. smooth, sharp). A smooth filter reduces spatial resolution as well as image noise; a sharp filter increases spatial resolution as well as image noise. The noise is caused by the fluctuations in the number of $\mathrm{X}$ ray quanta registered by the detector and is affected by convolution and dose. The SNR evaluates the ratio between the intensity of the signal and its statistical fluctuation. Suzuki et al. have demonstrated that the accuracy of a vascular diameter measurement with an automated software is affected by the choice of convolution filtering [27]. Seifarth et al. recently compared the effect of different convolution filtering on the visualization of coronary artery stents. Using the edge-enhancing b46f, they observed a significant improvement in lumen assessment compared to the conventional b30f [28].

Our study shows how the use of different convolution filters significantly affects the measured coronary plaque attenuation (Fig. 3). The use of sharper convolution filters produces an increase in the mean attenuation value of the calcified component of plaque. Increasingly sharper filtering produces a reduction in the mean attenuation values of the noncalcified plaque component. On the basis of our results, it is still challenging to rely on absolute HU values since the protocol used in coronary MSCT has not
Fig. 4 Example of plaque with increasing filtering using a saline and contrast solution

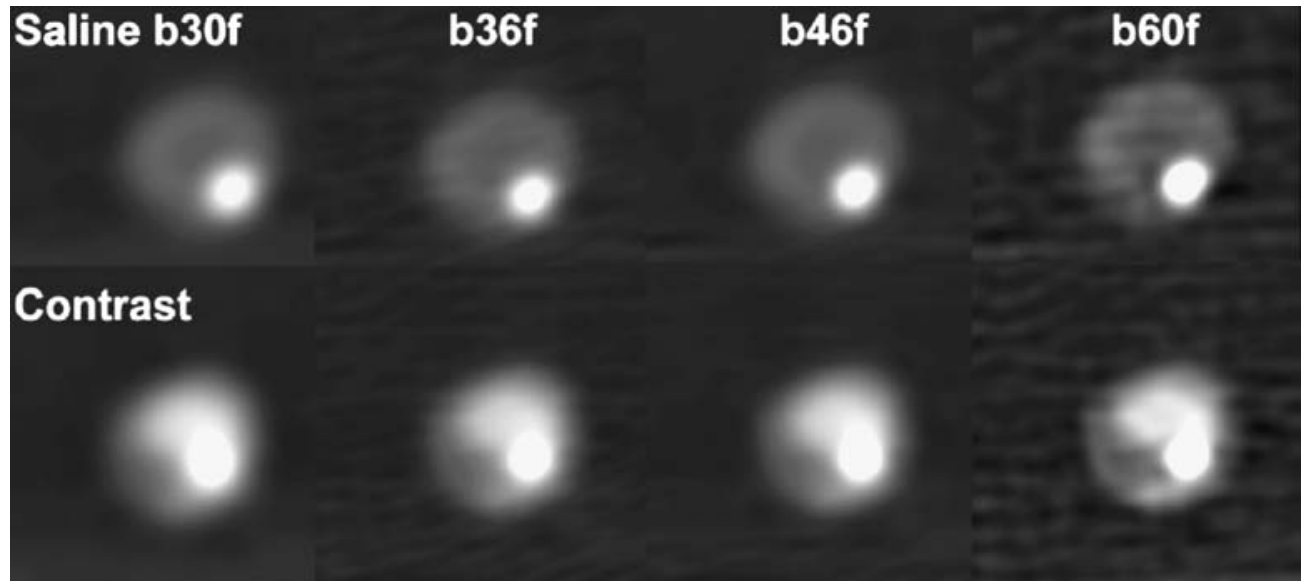


been standardized. Furthermore, absolute plaque attenuation values determined in the various studies are not comparable. It is reasonable to use a proper convolution filtering with improved SNR and CNR to better focus on plaque components. The b36f intermediate filter obtained a slight decrease of noncalcified plaque attenuation and an increase of calcified plaque attenuation. However, improved SNRs and CNRs were also achieved by the b30f and b46f filters. The b36f underestimated the noncalcified plaque attenuation and overestimated the calcified plaque attenuation in the likely attempt to weigh both components.

There are several limitations to our study. The first one is inherent to the lack of an evaluation of intra- and interobserver variability. A second limitation is related to the fact that the coronary specimens were collected from elderly patients with a high prevalence of calcification. The blooming artifacts of calcification may impair coronary noncalcified plaque visualization and characterization based on HU values [29]. Furthermore, we did not investigate the influence of $\mathrm{mAs}$ and voltage $(\mathrm{kV})$ on the blooming effect. Nevertheless, it has been reported that the voltage setting employed (i.e. $120 \mathrm{kV}$ ) might be reasonably chosen during in vivo studies [29]. In vivo studies should also better address the issue of body mass, which affects coronary plaque measurements [30]. Moreover, according to data mined from the literature, it is reasonable to expect that plaque size (especially in calcified plaques) and lumen area would be influenced by the use of different convolution filters due to variable partial volume effect. Edgeenhancing sharper reconstruction filters are reported to increase the visible in-stent lumen diameter [28, 31].

The pathological correlation has not been provided since the aim of our study was not to compare pathology with MSCT, but to assess the influence of convolution filtering on plaque attenuation measurement.

In conclusion, the use of different convolution filtering significantly modified plaque attenuation values. Therefore, convolution filtering should be reported when attenuation values are shown in order to provide a standardization of the methodology. Sharper convolution kernels increased the attenuation of the calcium within the coronary plaques and reduced the attenuation of soft plaque tissues. Improved SNR and CNR were achieved by using the b30f and b46f filters. A smooth filter (e.g. b30f) may be used for clinical routine evaluation, while a medium filter (e.g. b46f) may be considered the best choice for the assessment of highly calcified or stented vessels. The use of a proper filtering according to plaque type could give a more reliable assessment of plaque attenuation values in terms of HU.

\section{References}

1. Nieman K, Cademartiri F, Lemos PA, Raaijmakers R, Pattynama PM, de Feyter PJ (2002) Reliable noninvasive coronary angiography with fast submillimeter multislice spiral computed tomography. Circulation 106:2051-2054

2. Leschka S, Alkadhi H, Plass A, Desbiolles L, Grunenfelder J, Marincek B, Wildermuth S (2005) Accuracy of MSCT coronary angiography with 64slice technology: first experience. Eur Heart J 26:1482-1487

3. Raff GL, Gallagher MJ, O’Neill WW, Goldstein JA (2005) Diagnostic accuracy of noninvasive coronary angiography using 64-slice spiral computed tomography. J Am Coll Cardiol 46:552-557

4. Pugliese F, Mollet NR, Runza G, van Mieghem C, Meijboom WB, Malagutti P, Baks T, Krestin GP, Defeyter PJ, Cademartiri F (2005) Diagnostic accuracy of non-invasive 64-slice CT coronary angiography in patients with stable angina pectoris. Eur Radiol 1-8
5. Mollet NR, Cademartiri F, Nieman K, Saia F, Lemos PA, McFadden EP, Pattynama PM, Serruys PW, Krestin GP, de Feyter PJ (2004) Multislice spiral computed tomography coronary angiography in patients with stable angina pectoris. J Am Coll Cardiol 43:2265-2270

6. Hoffmann MH, Shi H, Schmitz BL, Schmid FT, Lieberknecht M, Schulze R, Ludwig B, Kroschel U, Jahnke N, Haerer W, Brambs HJ, Aschoff AJ (2005) Noninvasive coronary angiography with multislice computed tomography. Jama 293:2471-2478

7. Fuster V, Badimon L, Badimon JJ, Chesebro JH (1992) The pathogenesis of coronary artery disease and the acute coronary syndromes (1). N Engl J Med 326:242-250

8. Fuster V, Badimon L, Badimon JJ, Chesebro JH (1992) The pathogenesis of coronary artery disease and the acute coronary syndromes (2). N Engl J Med 326:310-318

9. Virmani R, Kolodgie FD, Burke AP, Farb A, Schwartz SM (2000) Lessons from sudden coronary death: a comprehensive morphological classification scheme for atherosclerotic lesions. Arterioscler Thromb Vasc Biol 20:1262-1275
10. Mintz GS, Nissen SE, Anderson WD, Bailey SR, Erbel R, Fitzgerald PJ, Pinto FJ, Rosenfield K, Siegel RJ, Tuzcu EM, Yock PG (2001) American College of Cardiology clinical expert consensus document on standards for acquisition, measurement and reporting of Intravascular Ultrasound Studies (IVUS). A report of the American College of

Cardiology task force on clinical expert consensus documents. J Am Coll Cardiol 37:1478-1492

11. Hatsukami TS, Ross R, Polissar NL, Yuan C (2000) Visualization of fibrous cap thickness and rupture in human atherosclerotic carotid plaque in vivo with high-resolution magnetic resonance imaging. Circulation 102:959-964

12. Fayad ZA, Fuster V, Fallon JT, Jayasundera T, Worthley SG, Helft G, Aguinaldo JG, Badimon JJ, Sharma SK (2000) Noninvasive in vivo human coronary artery lumen and wall imaging using black-blood magnetic resonance imaging. Circulation 102:506-510

13. Botnar RM, Stuber M, Kissinger KV, Kim WY, Spuentrup E, Manning WJ (2000) Noninvasive coronary vessel wall and plaque imaging with magnetic resonance imaging. Circulation 102:2582-2587 
14. Kopp AF, Schroeder S, Baumbach A, Kuettner A, Georg C, Ohnesorge B, Heuschmid M, Kuzo R, Claussen CD (2001) Non-invasive characterisation of coronary lesion morphology and composition by multislice $\mathrm{CT}$ : first results in comparison with intracoronary ultrasound. Eur Radiol 11:1607-1611

15. Schroeder S, Kopp AF, Baumbach A, Meisner C, Kuettner A, Georg C, Ohnesorge B, Herdeg C, Claussen CD, Karsch KR (2001) Noninvasive detection and evaluation of atherosclerotic coronary plaques with multislice computed tomography. J Am Coll Cardiol 37:1430-1435

16. Achenbach S, Moselewski F, Ropers D, Ferencik M, Hoffmann U, MacNeill B, Pohle K, Baum U, Anders K, Jang IK, Daniel WG, Brady TJ (2004) Detection of calcified and noncalcified coronary atherosclerotic plaque by contrast-enhanced, submillimeter multidetector spiral computed tomography: a segment-based comparison with intravascular ultrasound. Circulation 109:14-17

17. Leber AW, Knez A, Becker A, Becker C, von Ziegler F, Nikolaou K, Rist C, Reiser M, White C, Steinbeck G, Boekstegers P (2004) Accuracy of multidetector spiral computed tomography in identifying and differentiating the composition of coronary atherosclerotic plaques: a comparative study with intracoronary ultrasound. J Am Coll Cardiol 43:1241-1247

18. Leber AW, Becker A, Knez A, von Ziegler F, Sirol M, Nikolaou K, Ohnesorge B, Fayad ZA, Becker CR, Reiser M, Steinbeck G, Boekstegers P (2006) Accuracy of 64-slice computed tomography to classify and quantify plaque volumes in the proximal coronary system a comparative study using intravascular ultrasound. J Am Coll Cardiol 47:672-677, Feb 7
19. Leber AW, Knez A, von Ziegler F, Becker A, Nikolaou K, Paul S, Wintersperger B, Reiser M, Becker CR, Steinbeck G, Boekstegers P (2005) Quantification of obstructive and nonobstructive coronary lesions by 64 -slice computed tomography a comparative study with quantitative coronary angiography and intravascular ultrasound. J Am Coll Cardiol 46:147-154

20. Leber AW, Knez A, White CW, Becker A, von Ziegler F, Muehling O, Becker C, Reiser M, Steinbeck G, Boekstegers P (2003) Composition of coronary atherosclerotic plaques in patients with acute myocardial infarction and stable angina pectoris determined by contrast-enhanced multislice computed tomography. Am J Cardiol 91:714-718

21. Budoff MJ, Cohen MC, Garcia MJ, Hodgson JM, Hundley WG, Lima JA, Manning WJ, Pohost GM, Raggi PM, Rodgers GP, Rumberger JA, Taylor AJ, Creager MA, Hirshfeld JW Jr, Lorell BH, Merli G, Rodgers GP, Tracy CM, Weitz HH (2005) ACCF/AHA clinical competence statement on cardiac imaging with computed tomography and magnetic resonance: a report of the American College of Cardiology Foundation/ American Heart Association/American College of Physicians Task Force on Clinical Competence and Training. J Am Coll Cardiol 46:383-402

22. Becker CR, Nikolaou K, Muders M, Babaryka G, Crispin A, Schoepf UJ, Loehrs U, Reiser MF (2003) Ex vivo coronary atherosclerotic plaque characterization with multi-detector-row CT. Eur Radiol 13:2094-2098

23. Schroeder S, Flohr T, Kopp AF, Meisner C, Kuettner A, Herdeg C, Baumbach A, Ohnesorge B (2001) Accuracy of density measurements within plaques located in artificial coronary arteries by X-ray multislice CT: results of a phantom study. J Comput Assist Tomogr 25:900-906

24. Cademartiri F, Mollet NR, Runza G, Bruining N, Hamers R, Somers P, Knaapen M, Verheye S, Midiri M, Krestin GP, de Feyter PJ (2005) Influence of intracoronary attenuation on coronary plaque measurements using multislice computed tomography: observations in an ex vivo model of coronary computed tomography angiography. Eur Radiol 15:1426-1431
25. Flohr TG, McCollough $\mathrm{CH}$, Bruder $\mathrm{H}$, Petersilka M, Gruber K, Suss C, Grasruck M, Stierstorfer K, Krauss B, Raupach R, Primak AN, Kuttner A, Achenbach S, Becker C, Kopp A, Ohnesorge BM (2006) First performance evaluation of a dual-source CT (DSCT) system. Eur Radiol 16:256268

26. Johnson TR, Nikolaou K, Wintersperger BJ, Leber AW, von Ziegler F, Rist C, Buhmann S, Knez A, Reiser MF, Becker CR (2006) Dual-source CT cardiac imaging: initial experience. Eur Radiol 16:1409-1415

27. Suzuki S, Furui S, Kaminaga T, Yamauchi T (2004) Measurement of vascular diameter in vitro by automated software for CT angiography: effects of inner diameter, density of contrast medium, and convolution kernel. AJR Am J Roentgenol 182:1313-1317

28. Seifarth H, Raupach R, Schaller S, Fallenberg EM, Flohr T, Heindel W, Fischbach R, Maintz D (2005) Assessment of coronary artery stents using 16-slice MDCT angiography: evaluation of a dedicated reconstruction kernel and a noise reduction filter. Eur Radiol 15:721-726

29. de Weert TT, Ouhlous M, Zondervan PE, Hendriks JM, Dippel DW, van Sambeek MR, van der Lugt A (2005) In vitro characterization of atherosclerotic carotid plaque with multidetector computed tomography and histopathological correlation. Eur Radiol 15:1906-1914

30. Stanford W, Burns TL, Thompson BH, Witt JD, Lauer RM, Mahoney LT (2004) Influence of body size and section level on calcium phantom measurements at coronary artery calcium CT scanning. Radiology 230:198-205

31. Maintz D, Seifarth H, Flohr T, Kramer S, Wichter T, Heindel W, Fischbach R (2003) Improved coronary artery stent visualization and instent stenosis detection using 16-slice computed tomography and dedicated image reconstruction technique. Invest Radiol 38:790-795 